\title{
On the Influence of the Supine Posture on Simulation Sickness in Virtual Reality
}

\author{
Julien Marengo \\ Immersive Interaction Group \\ EPFL \\ Lausanne, Switzerland \\ julien.marengo@gmail.com
}

\author{
Phil Lopes \\ Immersive Interaction Group \\ EPFL \\ Lausanne, Switzerland \\ phil.lopes@epfl.ch
}

\author{
Ronan Boulic \\ Immersive Interaction Group \\ EPFL \\ Lausanne, Switzerland \\ ronan.boulic@epfl.ch
}

\begin{abstract}
Virtual Reality (VR) has the capability of fully immersing users into a wide variety of gaming experiences where the sole focus of the user is on that experience itself. One problem that often occurs when it comes to VR experiences is that of simulation sickness (SS), which is particularly prevalent when the user is forced to play at an abnormal posture due to physical limitations or specific treatment procedures. In this paper we report on a between group study comparing the effect of SS mitigating factors for two postures: seated and lying-down on one's back (known as the supine pose). A 3D recreation of the popular game Pacman (Namco, 1980) was developed specifically for VR. Each subject participated a total of six sessions of six minutes each taking place over the course of two to three weeks with a two to five day minimum and maximum between each session, respectively. At the start and end of each session participants were asked to fill-in the Simulator Sickness Questionnaire (SSQ), allowing to effectively rate the intensity of SS per session. Results show a lack of significant differences when examining participants as a whole regarding the SS decrease in relation with the field of view (FOV) and the rest frame factors. However, when considering the participant experience in VR, it appears that the lying-down pose does trigger simulation sickness symptoms for more proficient participants whereas they do not report such symptoms for the seated posture.
\end{abstract}

Index Terms-Virtual Reality, Immersive Video Game, Simulation Sickness, Rest Pose, Supine Pose

\section{INTRODUCTION}

Virtual Reality (VR) has the capability of fully immersing users into a wide variety of experiences, providing a fully interactive simulation where the sole focus of the user is on that experience itself. One problem that often occurs when it comes to VR experiences is that of simulation sickness (SS), which is particularly prevalent when the user is forced to play at an abnormal posture due to physical limitations or specific treatment procedures (i.e. lying-down on one's back, known as the supine pose). This can aggressively mitigate the users engagement by involuntarily inducing nausea forcing them to abandon the experience. This work intends to investigate which are the factors that contribute in decreasing the risk of SS according to different individual postures of play, such as seated and lying-down positions so that a set of characteristics

This work has been supported by the Hasler fundation with grant 18057 and partially supported by the SNF grant $C R S I I 5_{1} 80319 / 1$.

978-1-7281-1884-0/19/\$31.00 @2019 IEEE and factors can be learned and subsequently applied in systems that are intended to be used while the user is lying-down.

Even though SS has been a heavily researched topic in VR [1], there are still certain aspects that have rarely been touched upon, such as how posture can increase (or reduce) the risk of nausea. This work intends to study this risk and how the postures of play can contribute to the effect of $\mathrm{SS}$, specifically in a lying-down position. The experiment being targeted attempts to investigate the differences between the seated and lying-down postures, to understand if there is any substantial difference in SS intensity between them. Furthermore, to understand if VR experience could potentially be a factor in suppressing SS in both conditions, we explored if there is a difference between lower and medium-higher proficiency users. Lastly, habituation can also play a role in suppressing SS [2] and as such we observed each users motion sickness tolerance over a total of six sessions that took place over the course of two to three weeks. This experiment intends to understand the specific triggers that can directly induce SS in different posture positions, if training could potentially reduce this effect in individuals and eventually learning how to design scenarios capable of minimizing the risk of nausea while lying-down.

A recreation of the popular game Pacman (Namco, 1980) was developed specifically for VR, serving as the testbed for the experimental protocol similar to the work presented in [3]. Participants are tasked in playing the game for a total of six minutes in either the seated or lying-down posture positions. In addition to this, both groups are subjected to different SS mitigation techniques which are explored in the literature. Dynamic field of view (FOV) [4] allows the system to dynamically constrain the FOV based on user head movement. Another technique explored is the inclusion of static environmental markers in the environment (i.e. the sky), allowing participants to rest and stabilize themselves during play [5]. Participants in both the seated and lyingdown postures are subjected to all different conditions over the course of six different sessions to explore the habituation factor i.e. does SS intensity lower over the course of all six sessions? At the start and end of each session participants are asked to fill-in the Simulator Sickness Questionnaire (SSQ) [6], allowing to effectively rate the intensity of SS per session. 
The total score consists of calculating the difference between the post- and pre-experiment simulator sickness score for each session $(\Delta$ Score $)$.

We now examine the main related work in Virtual Reality in relation with the assessment of simulation sickness prior to summarize our contributions.

\section{RELATED WORK}

Since 2016 VR has been going through a renaissance due to devices such as the HTC Vive (HTC, 2016) and the Oculus Rift (Oculus, 2016) becoming substantially more affordable than ever before. This has also been true for the field of VR research, where it has seen an increase in activity over the past few years and has slowly been expanding into other areas of research. The application of VR in other fields of research has showcased that this medium can still present certain limitations that complicate its integration for certain types of experimentation. The most common difficulty is simulator sickness, which can ruin an experiment by cutting the participant experience short due to nausea inducing effects. Furthermore, this can be amplified due to the unorthodox postures that participants are forced to play-in, due to the data collection equipment that are sometimes used in these fields (e.g. fMRI) [7], [8]. Thus, this paper intends to explore the problem of simulator sickness by comparing a common posture (i.e. seated) with an uncommon posture (i.e. lyingdown) and its influence on simulator sickness.

This section will offer a brief overview of current notable work and how it is currently being applied in other fields such as neuroscience, psychology and medical applications. Lastly, this section touches upon the simulator sickness problem and this paper's contribution to this field.

\section{A. Virtual Reality for Experimentation}

One of the core advantages of VR applications is that it allows individuals to be fully immersed and focused into the experience at hand. The majority of VR devices also offer a diverging range of motion controllers allowing players to have a more interactive experience. Given these features VR has become an attractive methodology for research in human-based phenomena such as emotion elicitation [9], [10], psychology [11], [12], neuroscience [13] and even medical applications [14] providing experimenters with fully controllable simulations intended for treatment of certain conditions.

Human-based studies rely directly on human extracted data, either it being physiological (i.e. sensors or images) or questionnaires answered by the participants themselves. For the former, extracting data can be cumbersome and often invasive, which forces participants to stay in specific poses so as to avoid adding noise in the captured signal. Furthermore, these devices are often fragile and lack enough robustness to endure highly interactive experiences such as VR or digital games in general. However, this has not stopped researchers in continuing this pursuit [15] exploring several types of human collected signals including: Galvanic Skin Response (GSR) [16], [17], Electrocardiogram (ECG) [18] and Electroencephalogram (EEG) [18]; or imaging techniques like functional Magnetic Resonance Imaging (fMRI) [7], [8]. In particular, the latter can be quite problematic for VR applications due to the restricting nature of fMRI itself, where individuals cannot move their heads and torso, the space within the machine is quite limited, individuals have to play in a lying-down position and the entire duration of one session can last a minimum of 30 minutes. As such, VR experiences must accommodate these restrictions in such a way that it does not hinder the overall experience (i.e. should feel similar or as close to normal play as possible).

These constraints can also be problematic for individuals with temporary or permanent limited mobility, and during clinical treatments. Previous work has shown that VR has been successfully used as a distraction for the treatment of burn victims [14] to the point of complementing pharmaceutical analgesics to manage pain. Thus, it can be an important asset learning about the limitations of VR and how unorthodox postures can influence the overall player experience.

\section{B. Simulation Sickness}

Simulation sickness (SS) is a form of motion sickness, which does not necessarily require "true" motion but can be induced due to a VR headset's wider field of view (FOV) and an inaccurate representation of motion in the virtual simulated environment [19]. Similarly to motion sickness, SS can cause similar symptoms such as discomfort, nausea, sweating and rarely vomiting in individuals. Thus, SS has been a common problem and consequently heavily researched topic in the field of VR [19], [20], as it can aggressively mitigate the users engagement by involuntarily inducing nausea and forcing them to abandon the experience.

Previous work has attempted to pin-point different causes of increased risk of motion sickness in individuals, as the sensitivity to this condition can vary substantially among different users. One of the most common research trends in SS literature is the motion latency problem [21], where the dissonance between head-tracking motion and visual feedback can result in strong sensations of discomfort and possibly SS. Thus, as a general rule when designing VR software such situations should be avoided. Attempts to mitigate this problem have also been explored in previous work, such as reducing the FOV during head-tracking [4], by providing rest- or staticframes on screen such as cockpit views or reference points [5], [22], smoothing and blurring non-salient characteristics of the scene during certain motions [23] or even developing analytic software to facilitate the collection of SS data [24].

Even though simulator sickness has been a heavily researched topic, there are still certain aspects that have rarely been touched upon, such as when the user is forced to play in an abnormal posture (i.e. lying-down) due to physical limitations or specific treatment procedures. Considering the necessity of different usability cases of VR either for experimentation, clinical applications or entertainment exploring different methods of play could provide a valuable insight in the previously mentioned fields. Thus, this paper presents 


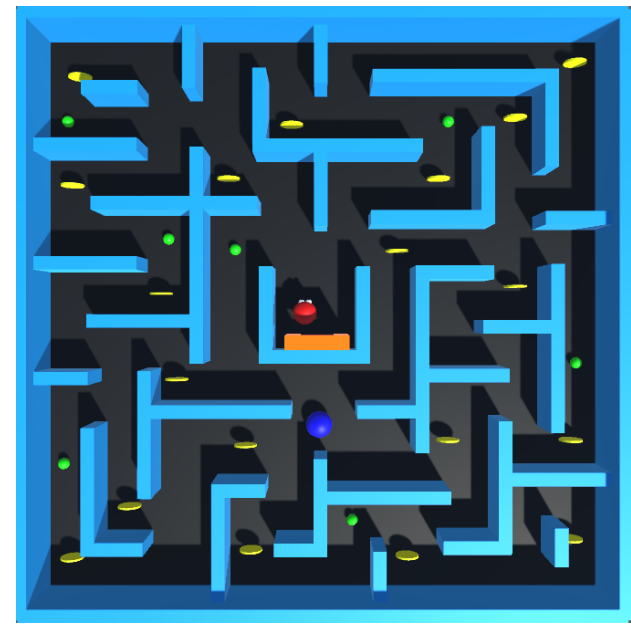

Fig. 1: Top-Down view of the static Pacman level used for experimentation. Yellow circles are the coins; green circles are "power-ups"; the red circle is the enemy ghost; the blue circle is the player; and the orange archway is the final portal and the ghost re-spawn area.

a preliminary study investigating the impact of SS in the lying-down position in comparison to the more popular seated position during several sessions of a VR version of Pacman (Namco, 1980), inspired by the work found in [3]. Lastly, SS is measured through the Simulation Sickness Questionnaire (SSQ) [6] the standard methodology for SS assessment.

\section{EXPERIMENTAl Methodology}

This section presents the overall details of the experiment such as the different conditions and variations of the Pacman game and the specific experimental protocol applied for experimentation.

\section{A. The Pacman Game}

The game itself is a simple version of a Pacman-like game where the level consists of a $25 \times 25 \mathrm{~m}^{2}$ (see Fig. 1). The player (blue circle) plays in a first person perspective where the goal is to collect all the coins (yellow circles) distributed among the labyrinth (19 in total) and reach the final portal (orange archway) that appears once all coins are collected. The player must navigate through the maze while avoiding the single ghost (red circle) that is constantly chasing them. If the ghost touches the player avatar, both characters restart in their initial positions with the player being penalized by losing half of their current collected coins. A total of 8 power-ups (green circles) are available to help players force the ghost to runaway in the opposite direction from them for a limited amount of time; it also allows the player to "eat" the ghost forcing it back to its initial position to "revive". Once a player completes a level by reaching the portal, the entire level resets with the ghost moving slightly faster then before $(+0.1 \mathrm{~m} / \mathrm{s})$. The ghost starts at a velocity of $1.15 \mathrm{~m} / \mathrm{s}$ and an angular velocity of 120 $\mathrm{deg} / \mathrm{s}$.

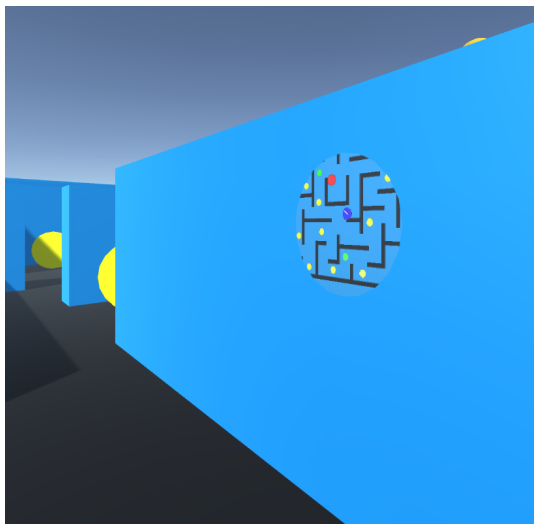

Fig. 2: Example of the mini-map being projected on one of the walls of the level. Includes all visible objects including: coins, power-ups, enemy, player and final portal; within a $20 \mathrm{~m}$ diameter.

The original Pacman is a 2-dimensional top-down game where players are able to visualize the entire maze, but once it is translated into a first person VR experience this information is significantly limited due to the players limited field of view constrained by the walls present in the maze. Thus, to help the players orient themselves within the maze a small mini-map (see Fig. 2) can be projected on any wall of the level with a touch of a button. The map showcases every object and walls within a $20 \mathrm{~m}$ diameter around the player including the enemy and the player.

The game itself is implemented in Unity 2017.3 and runs at an average 90 frames per second. The VR headset used consists of an Oculus Rift CVI allowing head tracking and Oculus Touch controller support. Players have direct control over their avatar by using the joysticks on the left touch controller to translate their character in four different directions: front, back, left and right. The maximum speed allowed for frontal movement is $2.94 \mathrm{~m} / \mathrm{s}$, while for all other directions the speed is cut in half. Players can rotate their avatar using the right joystick, where the angular speed is 50 degrees/s which was considered the most comfortable from our initial pilot studies. One group of participants also have the ability to use a teleportation mechanism in addition to the joystick controls, allowing them to point towards an intended location within the maze using one of the trigger buttons and instantly teleporting towards that location. However, due to the constraining nature of the maze and the sensitivity of the pointing mechanism this feature was rarely used in-game by participants.

Before starting each session players are put through a small training level to familiarize themselves with the concepts and mechanisms of the game. This small level contains a total of two coins, one power-up and a simplified enemy patrolling the same unique path. This level ends as soon as the participant reaches the final portal at the end of the path.

Lastly, to provide a full in-depth experience each participant wore a pair of Bose QuietComfort 35 wireless headphones, allowing subjects to be fully isolated from outside influences 


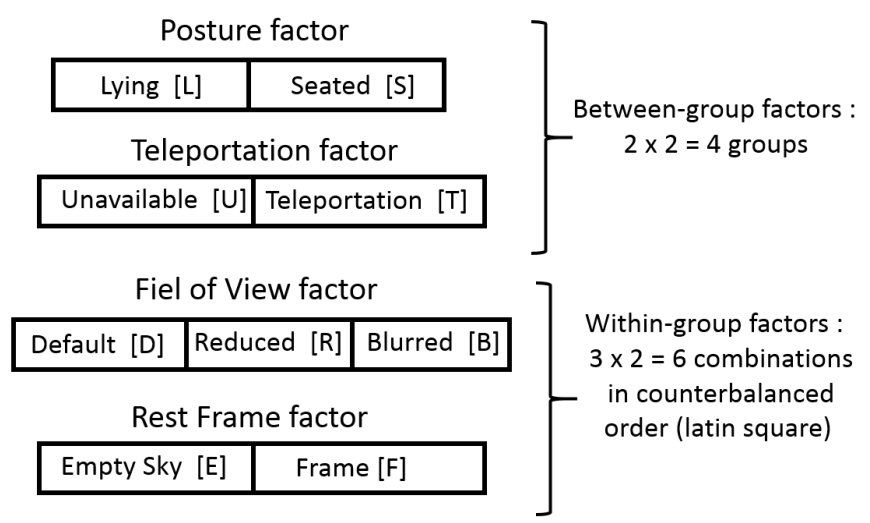

Fig. 3: Summary of the different factors and their respective group type.

due to the noise cancelling features offered by these headphones. A video demonstration of game-play can be found in the following $\operatorname{link} 1$.

\section{B. Posture}

The focus of this experiment was to investigate the risk of SS between two different playing postures: seated (S) and lying-down (L) (see Fig. 4). Participant groups and their respective factors were constructed using the Balanced Latin Square to ensure that there was a participant balance between the different factors being explored (see Fig. 3). Each participant was assigned a single posture position for the entirety of the experiment (i.e. for all 6 sessions). For each position the experimenters made sure that each participant was comfortable and safe during the experiment. Furthermore, it is important to note that participants in the $\mathrm{S}$ posture had an easier time moving their head, thus providing more degrees of freedom than the L position.

Each posture version of the Pacman game is similar. To achieve this the camera angle view was adjusted for the L version of the game (90 degrees). This way the exact same player perspective as the $\mathrm{S}$ version was achieved, while individuals were lying-down. Thus, when the user was lying-down on his back in the real-world they were actually standing-up in the virtual one recreating the same experience for both posture versions.

\section{Extended Navigation (Teleportation)}

Movement in VR can be one of the biggest factors of SS [21], thus this game variant intends to explore this concept a bit further for both posture positions. Thus, the last Pacman variation adds the teleportation ability to the game allowing players to teleport towards player observable locations of the map referred to as $\mathrm{U}$ and $\mathrm{T}$ in Fig. 3 The teleportation metaphor allowed to remotely adjust the orientation at the target location by exploiting the angular rotation position of the Oculus controller. It was felt that this feature would be

\footnotetext{
${ }_{1}^{1}$ https://youtu.be/2jQjLRNuyOk
}

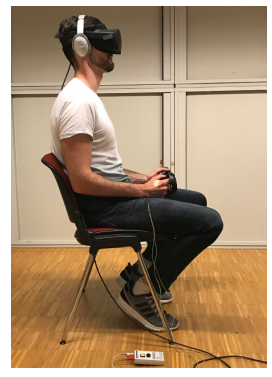

(a) The Seated (S) Position.

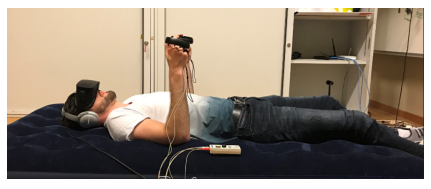

(b) The Lying-Down or Supine (L) Position.
Fig. 4: The different posture positions experimented.

appreciated in the relatively dense 3D labyrinth environment of the game as it allows to reduce the visual perception of rotation movement induced with the standard navigation metaphor. This was implemented as a complementary movement scheme to the joystick translation and was implemented using the VRTK [25] teleportation scripts. It is important to note that in this variation of the game players always have both controller schemes available at all times.

\section{Field of View (FOV)}

According to [4] reducing the FOV according to angular velocity of the head rotation and joystick rotation can reduce the risk of SS in VR games. Taking into account this study 3 variations of the FOV mechanisms were experimented in different sessions of the experiment. The FOV variations consists of:

- Default: Standard method with no FOV modification during angular head/joystick rotations.

- Reduced: A small black border appears around the screen during head/joystick rotations.

- Blurred: The screen border is blurred during head/joystick rotations.

Those variations are referred as D, R and B in Figure 3 The exact methodologies integrated within the context of this work can be found here [26]. Given that certain participants are lying-down during each play-session, head movement is significantly more restricted than the seated posture. As such, head movement in the seated position does not aggressively modify the players FOV due to their head angular speed, mitigating the differences existent between both posture positions.

\section{E. Rest Frame}

Rest frames have previously been suggested as a method of combating SS [5], [22], which can act as static references points within an environment to re-orient players within the virtual world. The core idea is to see whether adding a fixed background, such as a set of distant elements in the sky could potentially help reduce SS. Thus, two additional variations of the game were created: one version with no element in the sky (see Fig. 5-A) and another version with static orange spheres evenly distributed across the whole sky (see Fig. 5-B) (their subjective apparent size was slightly bigger than the sun while 


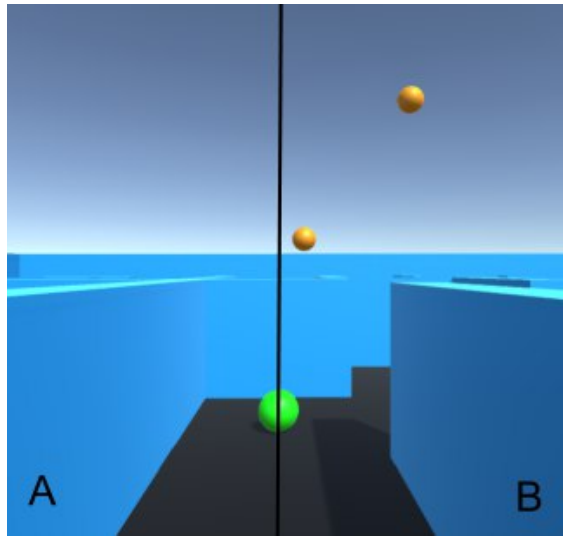

Fig. 5: The two different rest-frame conditions explored within this work. A: Empty sky condition without any orange spheres (without Resting Frames). B: Sky with uniformly distributed orange spheres (with Resting Frames).

their distribution ensured to always see two of them in the sky portion of the current field of view) referred as E and F on Figure 3

\section{F. Simulator Sickness Questionnaire (SSQ)}

The SSQ [6] has been a standard method to rate the intensity of simulation sickness within the community. It consists of a standard questionnaire (16 questions) that go over all the common symptoms that occur during SS. Symptoms include: General Discomfort, Fatigue, Headache, Eye Strain, Difficulty Focusing, Salivation Increasing, Sweating, Nausea, Difficulty Concentrating, "Fullness of the Head", Blurred Vision, Dizziness with eyes open, Dizziness with eyes closed, Vertigo, Stomach Awareness and Burping. For each symptom participants can rate the intensity on a 4-point scale ranging from: None, Slight, Moderate and Severe.

Calculating an SSQ score for each participant follows the standard method proposed by [6]. It consists of aggregating the value of each symptom based on one of three sub-classes: Oculomotor (eye related symptoms), Disorientation (dizziness, vertigo) and Nausea (stomach related symptoms). Each of these classes are multiplied by their respective weights such that:

$$
\begin{gathered}
N=T_{N s} * 9.54 \\
O=T_{O s} * 7.58 \\
D=T_{D s} * 13.92
\end{gathered}
$$

where $N, O, D$ are the respective scores for Nausea, Oculomotor and Disorientation, and $T_{N s}, T_{O s}$ and $T_{D s}$ are the aggregated totals of each symptomatic value associated to Nausea, Oculomotor and Disorientation, respectively. The final individual score (Score) is calculated by summing each sub-class and the respective weight as such:

$$
\text { Score }=N+O+D * 3.74
$$

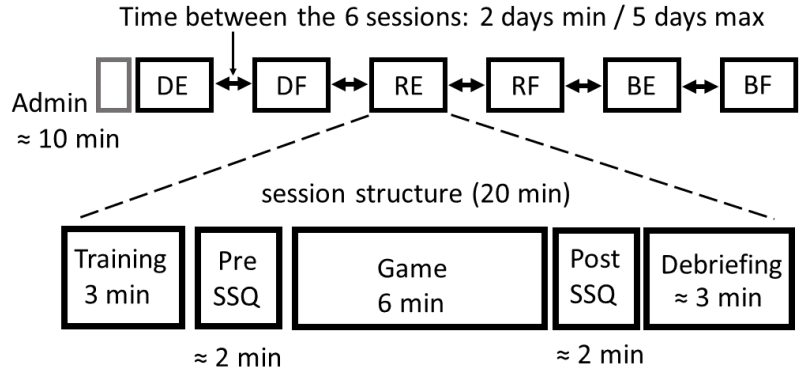

One session implements one combination of the within-group factors

Fig. 6: Example of one full participant experiment, consisting of a total of six sessions where the time between each session is over two to five days. Each session consists of a training phase (three minutes), the pre-SSQ (two minutes), the actual playing phase (six minutes), the post-SSQ and a debriefing session (three minutes). The only exception to the latter is the first session where an administration process (consent form, demographics and experimental briefing) is realized in addition to the other phases. Each playing segment varies based on the different factors being used.

SSQ scores are obtained before and after each individual playing session. Using both scores it is possible to calculate a $\Delta$ Score providing a more accurate representation of the overall SS intensity of the player for that particular session. Thus, $\Delta$ Score $_{i}$ can be calculated such that:

$$
\Delta \text { Score }_{i}=\text { ScorePost }_{i}-\text { ScorePre }_{i}
$$

where ScorePost $_{i}$ and ScorePre $_{i}$ are the respective SSQ scores after and before session $i$.

\section{G. Experimental Protocol}

For each individual participant an experiment consists of six sessions in total over the course of two to three weeks (with minimum two days and maximum five days between each session). The main reasoning is to test the potential factor of habituation in reducing the risk of SS. Each session lasts for a total of 20 minutes with the first session being the exception where its duration is 30 minutes due to administrative information procedure. During the first session participants are tasked in filling a consent form and a demographics survey (gender, VR experience, video game experience and frequency of play). Once completed players are informed of the rules of the game and its objectives.

Players are then given the VR headset and controllers. The first task consists of playing a small training level allowing them to get used to the different concepts and mechanics of the game, and can last for a total of 3 minutes. Once the training level is finished players are asked to fill the SSQ [6] to obtain an SSQ baseline score (pre-SSQ). Once completed the actual experimental play-session begins, lasting for a total of six minutes. The session finally ends with the participants 
filling the SSQ (post-SSQ), which is used in conjunction with the pre-SSQ to calculate the SSQ $\Delta$ Score.

Each session corresponds to a combination of factors which are balanced for each participants using the Balanced Latin Square method, i.e. 2 different rest frame variants crossed with 3 different FOV variants making a total of six sessions (see Fig. 6and 3). However, it was soon observed that teleportation was rarely or never being used during play by the sub-group with the capabilities to do so. This suggests that the differences between this sub-group and others are minimal at best due to the lack of usage whatsoever. For this reason we decided to focus on ensuring a balanced participation for the two posture groups without considering the additional subdivision linked to the navigation method.

\section{RESUlts}

This section presents the analysis obtained from the collected data over the course of experimentation. All experimental protocol code developed for the realization of this paper can be obtained in our public repository under the GNU General Public License 2 .

\section{A. Participants}

Demographics was collected from each participant to analyze the influence of playing proficiency in relation to the overall $\Delta S$ core for both posture groups. Information collected from each participant consisted of their gender and their VR experience (rated from 1 to 7). In total 33 subjects, aged from 18 to 31 years old, participated in the study (21 males and 12 females). Unfortunately not all participants were able to complete all sessions, where a total of 8 participants abandoned the experiment after the first session due to the intensity of SS (four females and four males). Thus, this data had to be discarded, where the total number of usable data obtained amounted to 25 participants (17 male and 8 female). Furthermore, participants were divided into two separate categories called "Low Proficiency" (rated VR experience between 1 and 2) and "Avg/High Proficiency" (rated VR experience 3 and above). This allowed to compare both groups evenly as the populations were as close to evenly distributed as possible: 16 and 9 participants for the Low and Avg/High proficiency, respectively.

\section{B. Game Variants for SS Mitigation}

In-depth analysis of the data shows that the different mitigation methods explored within this work to reduce the risk of SS were not particularly significant. Directly comparing the $\Delta S$ core of both seated and lying-down postures (see Fig. 7) showcases that no significant difference exists between these two groups. Even so, the distribution for the lying-down group indicates that some degree of participants did have a higher risk of simulation sickness.

Similar results were also found for the different variants of the game (i.e. FOV and Rest Frame), where no significant differences were observed. Thus, these results do suggest

\footnotetext{
${ }^{2}$ https://github.com/WorshipCookies/SupineSimulationSickness
}

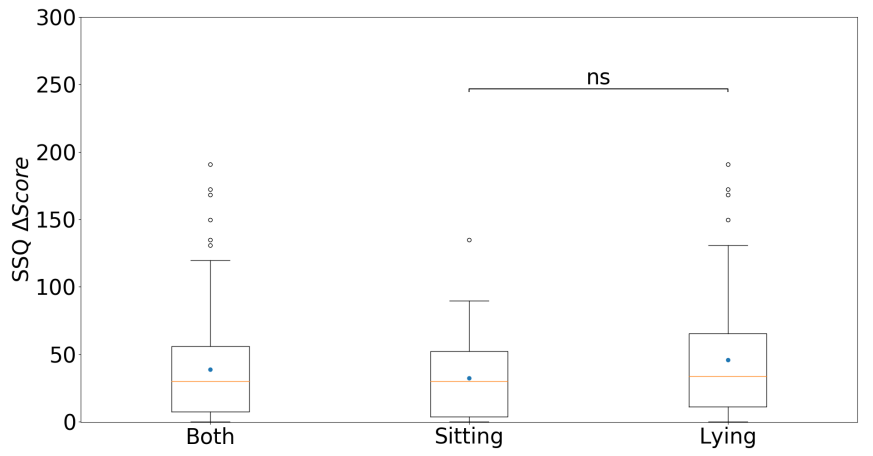

Fig. 7: Box-plot of the SSQ $\Delta$ Score for all participants and each posture groups. Yellow horizontal lines indicate the median, while blue dots indicate the mean. Using the KruskalWallis test no significant differences (ns) were observed between the two groups $(p<0.05)$.

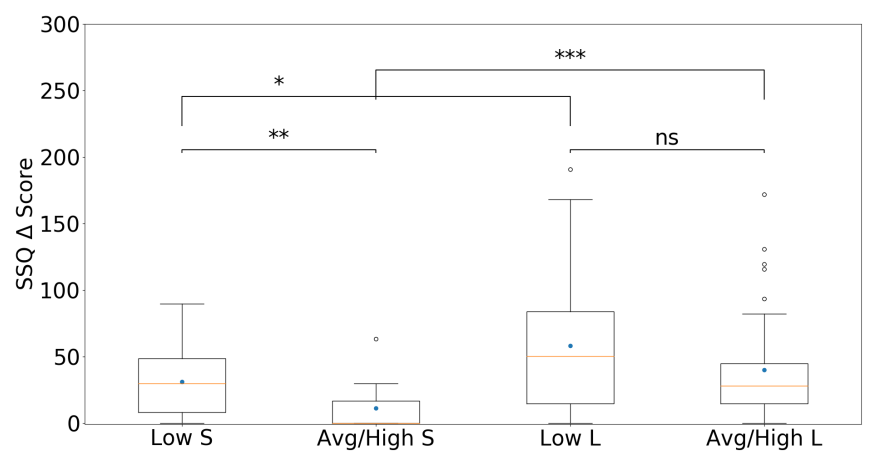

Fig. 8: Box-plot of the SSQ $\Delta$ Score for seated and lying-down groups and their respective proficiency. Yellow horizontal lines indicate the median, while blue dots indicate the mean. Significance was calculated using the Kruskal-Wallis test, where $(*)=p<0.05,(* *)=p<0.01$ and $(* * *)=p<0.001$

that these different SS mitigation techniques had little to no influence on either posture groups. Given this, for the purposes of space these latter results are omitted from the paper.

\section{Does VR Experience Influence SS?}

For this analysis each posture group was divided by proficiency, making sure that each proficiency sub-group was evenly split. Given that the majority of participants were in the "Low Proficiency" category (i.e. rated themselves between 1 and 2) this was kept as its own sub-group, while the remaining individuals were placed in the opposite sub-group dubbed "Average/High Proficiency" (i.e. rated themselves between 3 to 7). Figure 8 showcases the obtained $\Delta$ Score distributions obtained for each posture and proficiency group. Furthermore, the significant difference between these groups was also calculated using the Kruskal-Wallis test.

Thorough analysis suggests that there is a significant difference between the seated and lying-down postures from both proficiency groups. Although the difference is less prominent in the low proficiency group $(p<0.05)$, this result does make 


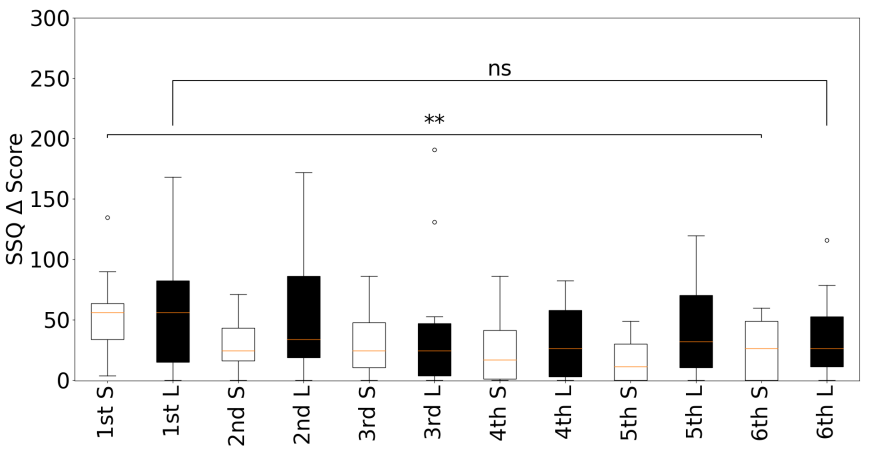

Fig. 9: Box-plot of the SSQ $\Delta S c o r e$ for Seated (White) and Lying-down (Black) groups over 6 experimental sessions. Significance between the first and final session was calculated using the Kruskal-Wallis test, where $(*)=p<0.05,(* *)=$ $p<0.01$ and $(* * *)=p<0.001$

sense considering that these participants have less experience and more prevalent to feeling sick even when seated. This theory is further reinforced due to the fact that more proficient participants presented a highly significant difference in the seated posture when compared to the less proficient ones $(p<0.01)$ using the same posture. Interestingly, the lyingdown posture seems to be difficult even for proficient players given the highly significant value obtained $(p<0.001)$. This suggests that even proficient individuals may find it difficult to experience VR when playing in a lying-down position.

\section{Does Habituation Diminish SS?}

Previous results suggest that player proficiency does have a certain degree of influence on the impact and risk of SS on individuals. This makes sense as more proficient individuals have a degree of habituation and "training", which could potentially decrease the risk of SS. Figure 9 showcases the statistic distribution of the SSQ $\Delta$ Score for both seated and lying-down groups over all six sessions. To clearly showcase the effects of habituation the Kruskal-Wallis significance test was calculated between the first and last sessions of both groups.

Results show that habituation did play a factor, however only for the seated posture where a significant difference was observed between the first and last sessions $(p<0.01)$. This further reinforces the prior results, where proficiency does not necessarily help mitigate the risk of SS specifically for the lying-down position. Although, analyzing this group on a per session basis, it can be seen that the distribution and variance does diminish as sessions pass, which may also suggest that this posture is a much harder playing position to get used to, but could potentially be possible over a longer period of time.

\section{DISCUSSION}

The obtained results suggest that even proficient individuals had difficulty in adjusting to the lying-down posture, where the risk of SS was still quite high for these types of participants. Furthermore, when analyzing the habituation factor it can be seen that individuals can get used to the seated posture over time, while for lying-down it can take significantly longer to get used to such an irregular position (if ever).

There are several hypothesis on why the lying-down position can prove so SS inducing, even for participants with significant VR training. First there is a clear dissonance between lying-down and walking upright in the virtual world, which can significantly confuse and disorient players during movement. Furthermore, given the abnormal posture it is highly probable that this was the first time participants (even proficient ones) attempted to play in such a posture. Normally, VR is experienced either seated or standing-up, thus individuals with enough experience can already be accustomed to the more popular postures, but once attempting to play lyingdown it has the potential of completely changing the experience. Lastly, when lying-down head rotation is significantly limited in comparison to the seated position, which forces the individuals to use the joystick to effectively explore their environments. This posture-induced constraint while lyingdown may have resulted in a greater use of the joystick while navigating in the labyrinth, hence amplifying the perceptual visuo-vestibular conflict at the root of most SS.

The difficulty in getting used to the lying-down posture is quite prominent in the results obtained over the 6 different sessions. Comparatively to the seated posture it is quite apparent that this posture was easier to get used to than lyingdown. These results agree with previous research in literature that habituation does help individuals handle SS better [2]. Even though the results were not significant for the first and the last sessions of the lying-down position, the trend of the distributions over time do suggest that given enough training individuals may be able to get used to this posture eventually, but as observed it may prove significantly more difficult than the other postures.

Reducing the risk of SS in a lying-down position could potentially be possible, although it might be a design problem specifically. This work specifically explored this problem within the context of a Pacman game, which requires player movement and translation. However, if a game is designed with the lying-down position in mind, where the player is not forced to make significant movements or even walk upright, it could potentially reduce the risk of SS. This is already true in several commercial VR games that do take into account the posture of the individual during play. Games such as Beat Saber (Beat Games, 2018) expect players to be standing and moving around, while a game such as Elite Dangerous $V R$ (Frontier Developments, 2016) players sit within a virtual cockpit traveling through space.

\section{CONCLUSION}

The purpose of this study was at first to evaluate the effect of different visual factors as well as postures on SS. Although our results were not the ones expected at first regarding our different visual factors, we found interesting relations about the supine lying-down position that is very rarely treated toward SS. Indeed, proficient participants were less inclined to 
suffer from SS in seated posture due to implicit training. On the other hand, since their past experience is based on standing/seated and not lying-down position, i.e. a different unusual position, their proficiency did not help them to handle SS in the lying-down position. Furthermore, we were able to confirm some statements about habituation in VR environment. Given these findings about the rest pose in Virtual Reality our future work involving game-like interaction in an fMRI device will be designed with the central requirement of being consistent with the lying-down posture to prevent the visuo-vestibular perceptual conflict. We expect this approach to be beneficial for other classes of potential VR users who are constrained to by lying-down (e.g. patients).

\section{ACKNOWLEDGMENT}

We would like to thank Thibault Porssut for the great help and availability he offered us for the implementation of the game. We would also like to thank all the participants that gracefully offered their time as pilot participant for this experiment.

\section{REFERENCES}

[1] Sue VG Cobb, Sarah Nichols, Amanda Ramsey, and John R Wilson. Virtual reality-induced symptoms and effects (vrise). Presence: Teleoperators \& Virtual Environments, 8(2):169-186, 1999.

[2] Alireza Mazloumi Gavgani, Keith V Nesbitt, Karen L Blackmore, and Eugene Nalivaiko. Profiling subjective symptoms and autonomic changes associated with cybersickness. Autonomic Neuroscience: Basic and Clinical, 203:41-50, 2017.

[3] Daniel Clarke, Graham McGregor, Brianna Rubin, Jonathan Stanford, and TC Graham. Arcaid: Addressing situation awareness and simulator sickness in a virtual reality pac-man game. In Proceedings of the 2016 Annual Symposium on Computer-Human Interaction in Play Companion Extended Abstracts, pages 39-45. ACM, 2016.

[4] Ajoy S Fernandes and Steven K Feiner. Combating vr sickness through subtle dynamic field-of-view modification. In 2016 IEEE Symposium on 3D User Interfaces (3DUI), pages 201-210. IEEE, 2016.

[5] Zekun Cao, Jason Jerald, and Regis Kopper. Visually-induced motion sickness reduction via static and dynamic rest frames. In 2018 IEEE Conference on Virtual Reality and $3 D$ User Interfaces (VR), pages 105112. IEEE, 2018.

[6] Robert S Kennedy, Norman E Lane, Kevin S Berbaum, and Michael G Lilienthal. Simulator sickness questionnaire: An enhanced method for quantifying simulator sickness. The international journal of aviation psychology, 3(3):203-220, 1993.

[7] Daniel S Pine, Joseph Grun, Eleanor A Maguire, Neil Burgess, Eric Zarahn, Vivian Koda, Abby Fyer, Philip R Szeszko, and Robert M Bilder. Neurodevelopmental aspects of spatial navigation: a virtual reality fmri study. Neuroimage, 15(2):396-406, 2002.

[8] Hunter G Hoffman, Todd Richards, Barbara Coda, Anne Richards, and Sam R Sharar. The illusion of presence in immersive virtual reality during an fmri brain scan. CyberPsychology \& Behavior, 6(2):127-131, 2003.

[9] Anna Felnhofer, Oswald D Kothgassner, Mareike Schmidt, AnnaKatharina Heinzle, Leon Beutl, Helmut Hlavacs, and Ilse Kryspin-Exner. Is virtual reality emotionally arousing? investigating five emotion inducing virtual park scenarios. International journal of human-computer studies, 82:48-56, 2015.

[10] Ben Meuleman and David Rudsrauf. Induction and profiling of strong multi-componential emotions in virtual reality. IEEE Transactions on Affective Computing, 2018.

[11] Sergi Bermudez i Badia, Luis Velez Quintero, Monica S Cameirao, Alice Chirico, Stefano Triberti, Pietro Cipresso, and Andrea Gaggioli. Towards emotionally-adaptive virtual reality for mental health applications. IEEE journal of biomedical and health informatics, 2018.
[12] Albert Rizzo, Judith Cukor, Maryrose Gerardi, Stephanie Alley, Chris Reist, Mike Roy, Barbara O Rothbaum, and JoAnn Difede. Virtual reality exposure for ptsd due to military combat and terrorist attacks. Journal of Contemporary Psychotherapy, 45(4):255-264, 2015.

[13] Jascha Grübel, Raphael P Weibel, Christoph Hölscher, and Victor Schinazi. Eve: A framework for running virtual reality experiments in neuroscience. In 2nd Interdisciplinary Navigation Symposium (iNAV 2018), page 100, 2018.

[14] Hunter G Hoffman, Gloria T Chambers, Walter J Meyer III, Lisa L Arceneaux, William J Russell, Eric J Seibel, Todd L Richards, Sam R Sharar, and David R Patterson. Virtual reality as an adjunctive nonpharmacologic analgesic for acute burn pain during medical procedures. Annals of Behavioral Medicine, 41(2):183-191, 2011.

[15] J Matias Kivikangas, Guillaume Chanel, Ben Cowley, Inger Ekman, Mikko Salminen, Simo Järvelä, and Niklas Ravaja. A review of the use of psychophysiological methods in game research. journal of gaming \& virtual worlds, 3(3):181-199, 2011

[16] Christoffer Holmgård, Georgios N Yannakakis, Héctor P Martínez, Karen-Inge Karstoft, and Henrik Steen Andersen. Multimodal ptsd characterization via the startlemart game. Journal on Multimodal User Interfaces, 9(1):3-15, 2015

[17] Guillaume Chanel, Cyril Rebetez, Mireille Bétrancourt, and Thierry Pun. Emotion assessment from physiological signals for adaptation of game difficulty. IEEE Transactions on Systems, Man, and Cybernetics-Part A: Systems and Humans, 41(6):1052-1063, 2011.

[18] Javier Marín-Morales, Juan Luis Higuera-Trujillo, Alberto Greco, Jaime Guixeres, Carmen Llinares, Enzo Pasquale Scilingo, Mariano Alcañiz, and Gaetano Valenza. Affective computing in virtual reality: emotion recognition from brain and heartbeat dynamics using wearable sensors. Scientific reports, 8(1):13657, 2018.

[19] David M Johnson. Introduction to and review of simulator sickness research. Technical report, U.S Army Research Institute for Behavioral and Social Sciences, Fort Rucker, Alabama, 2005.

[20] Sarah Sharples, Sue Cobb, Amanda Moody, and John R Wilson. Virtual reality induced symptoms and effects (vrise): Comparison of head mounted display (hmd), desktop and projection display systems. Displays, 29(2):58-69, 2008

[21] Jason J Jerald. Scene-motion-and latency-perception thresholds for headmounted displays. Doctor of Philosophy in the Department of Computer Science, University of North Carolina at Chapel Hill, 2010.

[22] Andras Kemeny, Paul George, Frédéric Mérienne, and Florent Colombet. New vr navigation techniques to reduce cybersickness. Electronic Imaging, 2017(3):48-53, 2017.

[23] Guangyu Nie, Yue Liu, and Yongtian Wang. [poster] prevention of visually induced motion sickness based on dynamic real-time contentaware non-salient area blurring. In 2017 IEEE International Symposium on Mixed and Augmented Reality (ISMAR-Adjunct), pages 75-78. IEEE, 2017.

[24] Sangho Lee, Ashley Koo, and Junekyo Jhung. Moskit: Motion sickness analysis platform for vr games. In 2017 IEEE International Conference on Consumer Electronics (ICCE), pages 17-18. IEEE, 2017.

[25] $\mathrm{Vr}$ toolkit. https://vrtoolkit.readme.io/docs Accessed: 2019-05-21.

[26] Unity vr tunelling project. https://github.com/SixWays/UnityVrTunnelling Accessed: 2019-05-21. 